\title{
A DOA Estimation Algorithm without Source Number Estimation for Nonplanar Array with Arbitrary Geometry
}

\author{
He Ke, Zhang Xiaomin, Han Peng, Yu Yang and Yan Shi
}

\begin{abstract}
The performances of most of the high resolution methods always depend on the estimation of the source number. In real application, when the estimated number of signals is not correct, the orthogonality between signal subspace and noise subspace can not be maintained any more. And the performance of DOA estimation algorithm will deteriorate severely. In this paper, a high resolution algorithm called $\mathbf{m}$ MVM without source number estimation and eigendecomposition for Direction-Of-Arrival (DOA) estimation is proposed, which is an improvement of the Minimum Variance Method (MVM). Furthermore, it is suitable to nonplanar arrays of arbitrary geometries. Some representative computer simulations are presented to illustrate the performance comparison between different algorithms and different arrays.
\end{abstract}

Index Terms-nonplanar array, DOA, MVM

\section{INTRODUCTION}

In recent decades, the problem of estimating signal parameters from data collected by an array of sensors has received much attention. And a great variety of highresolution algorithms for source localization [1,2] have been reported in the literature. Although they have superior accuracy and resolution performance over conventional beamforming techniques, most high-resolution algorithms depend on the source number estimation. Indeed, for those methods, we must truncate the covariance matrix in signal and noise subspaces. That truncation depends on the estimation of the source number. In real application, when the number of signals is underestimated or overestimated, the orthogonality between signal subspace and noise subspace can not be maintained any more. And the performance of DOA estimation algorithm will deteriorate severely. In [3,4], the performance of MUSIC algorithm under errors of source number is analyzed, and the proposed algorithms based on the eigendecomposition of sample covariance matrix can estimate DOA properly when the source number is unknown.

Although the high-resolution algorithms reported in the literature have demonstrated varying degrees of success, their applications are limited. In many situations, planar array can't meet the requirements of many actual detection systems. While nonplanar array mounted on the planes, bombs, ships, mines, and underwater vehicles will bring

He Ke, Zhang Xiaomin, Han Peng, Yu Yang are with College Of Marine, Northwestern Polytechnical University, Xi'an, China (email:hk@nwpu.edu.cn).

Yan Shi is with the Delegation of Navy at Kunming,Kunming, China (email: 13888910546@139.com). many benefits. However, few algorithms have been developed for this problem. And it is very important for the design and evaluation of nonplanar array to develop highresolution algorithm for nonplanar array.

In this paper, a nonplanar array with arbitrary geometry is modeled; we present a high-resolution algorithm based on the Minimum Variance Method (MVM) without source number estimation and eigendecomposition. Computer simulation for the performance comparison between the proposed algorithm and the MUSIC algorithm is demonstrated. And some conclusions of the proposed algorithm for the nonplanar quaternion array and planar array are reached.

\section{ARRAY DATA MODEL}

In this section, a model of arbitrary geometry is described for the nonplanar array. The array is assumed to be melement arbitrary nonplanar geometry, and $\mathrm{n}$ narrowband signals with known centre frequency from far field, as shown in Fig 1.

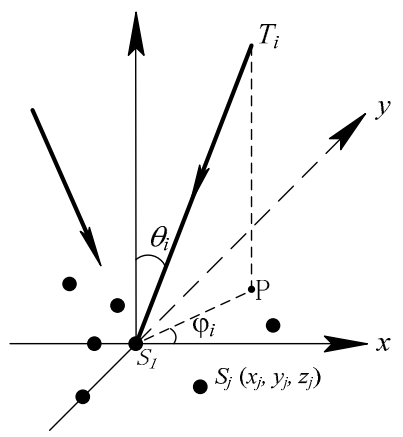

Figure 1. The nonplanar array with arbitrary geometry.

With the sensor S1 at the origin of the coordinate as reference, the array output can be written as

$$
X(t)=A \cdot S(t)+N(t)
$$

Where $X(t)=\left[x_{1}(t), x_{2}(t), \cdots, x_{m}(t)\right]^{T}$ represents a data vector, $S(t)=\left[s_{1}(t), s_{2}(t), \cdots, s_{n}(t)\right]^{T}$ the source vector, and $N(t)=\left[n_{1}(t), n_{2}(t), \cdots, n_{m}(t)\right]^{T}$ the noise vector.

The $\mathrm{i}^{\text {th }}$ column of the array manifold matrix $A$, which is represented by $a\left(\theta_{i}, \varphi_{i}\right)(i=1,2, \ldots n)$, is the steering vector for the $\mathrm{i}^{\text {th }}$ source signal, and is given by

$$
a\left(\theta_{i}, \varphi_{i}\right)=\left[e^{j 2 \pi f_{i} \tau_{i 1}}, e^{j 2 \pi f_{i} \tau_{i 2}} \cdots, e^{j 2 \pi f_{i} \tau_{i m}}\right]^{T}
$$

And $\tau_{i j}(j=1,2, \ldots m)$ is the differential time delay of the $i^{\text {th }}$ source signal between the $\mathrm{j}^{\text {th }}$ sensor and the coordinate reference 


$$
\begin{aligned}
& \tau_{\mathrm{ij}}=-\frac{u_{i} p_{j}^{T}}{c} \\
& =-\frac{1}{c}\left(x_{j} \cdot \sin \theta_{i} \cdot \cos \varphi_{i}+y_{j} \cdot \sin \theta_{i} \cdot \sin \varphi_{i}+z_{j} \cdot \cos \theta_{i}\right)
\end{aligned}
$$

Where $f_{i}$ is the centre frequency of the $i^{\text {th }}$ source signal and $\mathrm{c}$ is the speed of propagation in the medium of interest; $\theta_{i}$ and $\varphi_{\mathrm{i}}$ are the elevation angle and azimuth angle for the $\mathrm{i}^{\text {th }}$ source signal and $u_{i}=\left[\sin \theta_{i} \cdot \cos \varphi_{i}, \sin \theta_{i} \cdot \sin \varphi_{i}, \cos \theta_{i}\right]$ is the direction unit vector; $p_{j}=\left[x_{j}, y_{j}, z_{j}\right](j=1,2, \cdots m)$ is the vector made up by element of the coordinate.

With the assumption that signal sources are incoherent and noises are independent with zero mean and $\sigma_{n}{ }^{2}$ variance, the spatial covariance matrix of $X(t)$ is

$$
\begin{aligned}
& R=E\left[X X^{H}\right]=A E\left[S S^{H}\right] A^{H}+E\left[N N^{H}\right] \\
& =A R_{S} A^{H}+\sigma^{2} I
\end{aligned}
$$

Decompose the spatial covariance matrix $R$, the eigenvectors corresponding to the $N$ largest eigenvalues $\lambda_{i}$ and the rest are named as signal space $U_{S}=\left[e_{1}, e_{2}, \cdots, e_{N}\right]$ and noise space $U_{N}=\left[e_{N+1}, e_{N+2}, \cdots, e_{M}\right]$ respectively. According to the eigenstructure method, we obtain

$$
\begin{aligned}
& R=\sum_{i=1}^{N} \lambda_{i} e_{i} e_{i}^{H}+\sigma^{2} \sum_{j=N+1}^{M} e_{j} e_{j}^{H} \\
& =U_{S} \Sigma_{S} U_{S}^{H}+\sigma^{2} U_{N} U_{N}^{H}
\end{aligned}
$$

In practice, this matrix is estimated using $L$ snapshots of the actual array output, as shown in equ. 6

$$
\hat{R}=\frac{1}{L} \sum_{i=1}^{L} X X^{H}
$$

\section{ALGORITHM}

\section{A. MUSIC Algorithm}

With the orthogonality of the signal and noise subspaces, The MUSIC spectrum estimate is given by

$$
P_{\text {MUSIC }}(\theta, \varphi)=\frac{1}{\alpha^{H}(\theta, \varphi) U_{N} U_{N}^{H} \alpha(\theta, \varphi)}
$$

The estimation of $\theta$ and $\varphi$ is corresponding to the peaks of the MUSIC spectrum :

$$
\begin{aligned}
& (\hat{\theta}, \hat{\varphi})=\arg \max _{(\hat{\theta}, \hat{\varphi})}\left\{P_{M U S I C}(\theta, \varphi)\right\} \\
& =\arg \min _{(\hat{\theta}, \hat{\varphi})}\left\{\alpha^{H}(\theta, \varphi) U_{N} U_{N}^{H} \alpha(\theta, \varphi)\right\}
\end{aligned}
$$

However, the MUSIC Algorithm is sensitive to different noise fields and the performance is degraded in short snapshots.

\section{B. Minimum Variance Method}

Based on the minimum variance method (MVM), the MVM spectrum estimate is given by

$$
P_{M V M}(\theta, \varphi)=\frac{1}{\alpha^{H}(\theta, \varphi) R^{-1} \alpha(\theta, \varphi)}
$$

The estimation of $\theta$ and $\varphi$ is corresponding to the peaks of the MVM spectrum :

$$
\begin{aligned}
& (\hat{\theta}, \hat{\varphi})=\arg \max _{(\hat{\theta}, \hat{\varphi})}\left\{P_{M V M}(\theta, \varphi)\right\} \\
& =\arg \min _{(\hat{\theta}, \hat{\varphi})}\left\{\alpha^{H}(\theta, \varphi) R^{-1} \alpha(\theta, \varphi)\right\}
\end{aligned}
$$

The MVM spectrum is the maximum likelihood estimate of $\theta$ and $\varphi$, and is proportional to the power spectral of the signal. Comparing with the MUSIC method, it shows the advantage that MVM does not require source number estimation and eigendecomposition of sample covariance matrix. However, due to the array aperture limitation, MVM is usually at lower resolution.

\section{C. m-MVM Algorithm}

Combining the advantages of MUSIC and MVM, we can get better resolution without source number estimation and eigendecomposition. It is called m-MVM Algorithm, which is an improvement on the traditional MVM.

Transform the equ.5:

$$
\sigma^{2 m} R^{-m}=U_{S} \operatorname{diag}\left\{\left(\frac{\sigma^{2}}{\lambda_{i}}\right)^{m}\right\}^{2} U_{S}^{H}+U_{N} U_{N}^{H}
$$

Where $\mathrm{m}$ is an integer bigger than 2; Apparently, $\sigma^{2} / \lambda_{i}$ is less than 1 , so equ.11 approaches to noise space, as the $\mathrm{m}$ approaches to infinity.

$$
\lim _{m \rightarrow \infty} \sigma^{2 m} R^{-m}=U_{N} U_{N}^{H}
$$

As a matter of fact, the experiment shows that the performances of the algorithm are improved, when $\mathrm{m}$ is bigger than 2 .

So, the m-MVM algorithm can be expressed as:

$$
\begin{aligned}
& (\hat{\theta}, \hat{\varphi})=\arg \max _{(\hat{\theta}, \hat{\varphi})}\left\{\prod_{i=1}^{m} \frac{1}{\alpha^{H}(\theta, \varphi) \hat{R}^{-1} \alpha(\theta, \varphi)}\right\} \\
& =\arg \max _{(\hat{\theta}, \hat{\varphi})}\left\{\frac{1}{\alpha^{H}(\theta, \varphi) \hat{R}^{-m} \alpha(\theta, \varphi)}\right\} \\
& =\arg \min _{(\hat{\theta}, \hat{\varphi})}\left\{\alpha^{H}(\theta, \varphi) \hat{R}^{-m} \alpha(\theta, \varphi)\right\}
\end{aligned}
$$

For nonplanar array with arbitrary geometry, the m-MVM algorithm can be implemented in steps as following:

1) Set the parameters of the nonplanar array and the arrival angle, and set up the steering vector from equation (2) and (3), the array manifold matrix A can be derived.

2) Create the source signal data and random noise data using mathematical modeling, and calculate the data vector $X(t)$ from equation (1).

3) Calculate the correlation matrix $\mathrm{R}$ of the output of array from equation (6).

4) Calculate the $m-M V M$ spectrum using the steering vector and the correlation matrix with the following function.

$$
P_{m-M V M}(\theta, \varphi)=\frac{1}{\alpha^{H}(\theta, \varphi) R^{-m} \alpha(\theta, \varphi)}
$$

The physical meaning of the m-MVM algorithm is a connection of $\mathrm{m}$ MVM estimators. When $\mathrm{m}$ is equal to 1 , the $\mathrm{m}-\mathrm{MVM}$ algorithm is equal to MVM estimator. When approaches to infinity, the m-MVM algorithm approaches to 
MUSIC algorithm.

\section{PERFormanCE SimUlation}

In this section, the performance of the proposed algorithm is evaluated by some representative computer simulations. Numerical examples are carried out using two kind of array of 4 elements, as shown in figure 2.

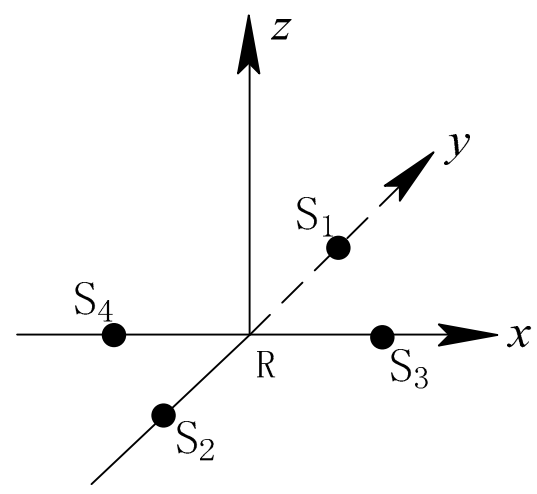

a. uniform cross array

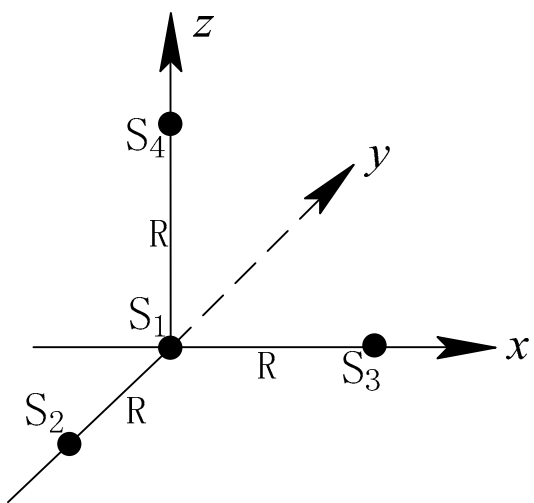

b. nonplanar quaternion array

Figure 2. The geometry of array

The assumed array is that of the uniform cross array and the nonplanar quaternion array with an intersensor spacing of $\lambda / 2$, where $\lambda$ is the wavelength of the source signals. The number of snapshots is fixed at 512 and two sources with the signal to noise ratio(SNR) of $10 \mathrm{~dB}$ arriving at $(45,-60)$ and $(30,30)$, which is the elevation angle and azimuth angle of the sources. The results of simulation are shown from Figure 3 to Figure 7. Figure 3 is the DOA estimation result using MUSIC algorithm with correct source number estimation, while Figure 4 is the MUSIC spectrum with wrong source number estimation. Notice that the MUSIC algorithm can not estimate the DOA with wrong source number estimation.

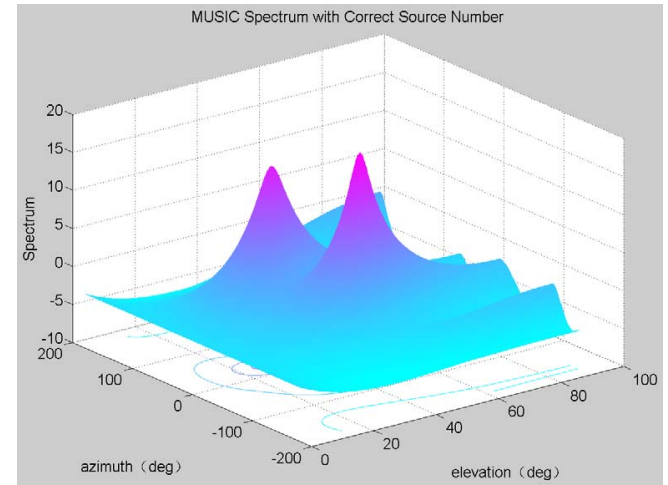

Figure 3. The MUSIC spectrum with correct source number

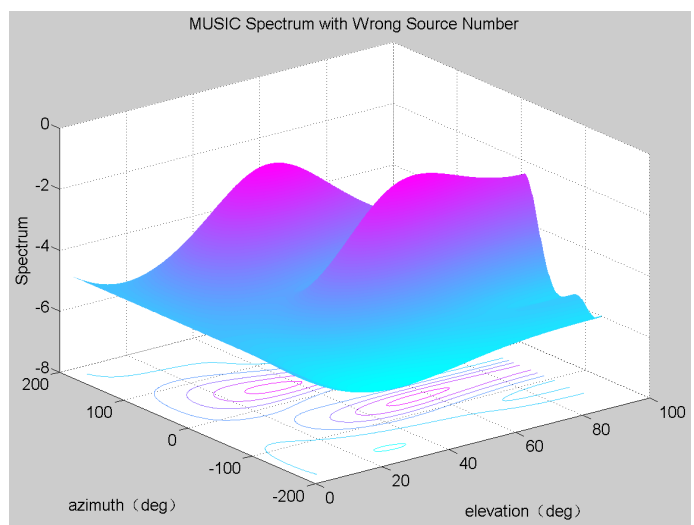

Figure 4. The MUSIC spectrum with wrong source number

Figure 5 and Figure 6 is the DOA estimation result using m-MVM algorithm without source number estimation for the nonplanar array and the uniform cross array. Notice that the performance of the m-MVM spectrum is better than the MUSIC spectrum, and the performance of the nonplanar array is better than the uniform cross array.

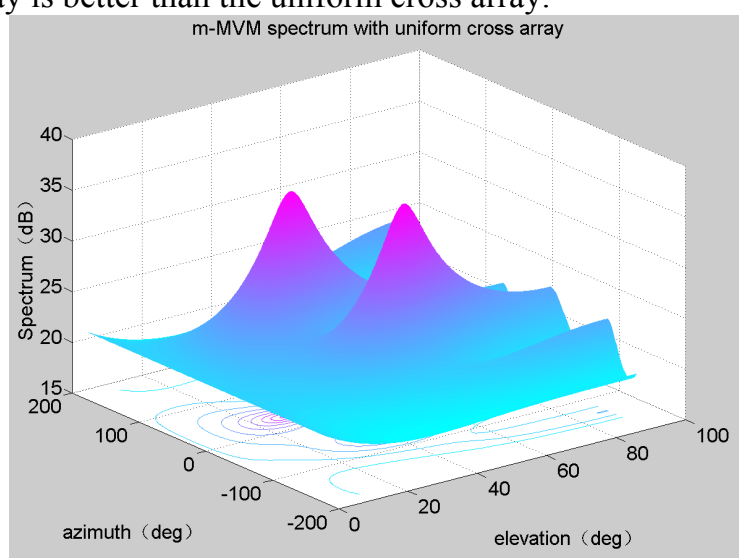

Figure 5. m-MVM spectrum for two sources with the uniform cross array 


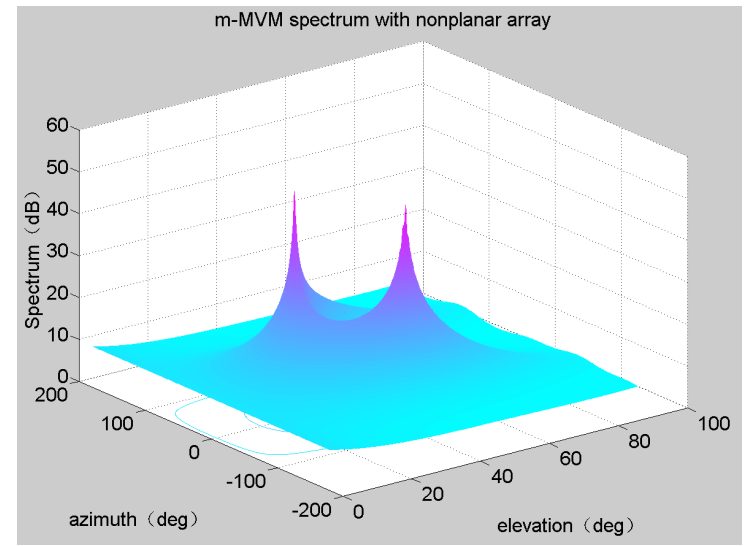

Figure 6. m-MVM spectrum for two sources with the nonplanar array

Figure 7 and Figure 8 show the deviation and variance of the m-MVM algorithm by Monte Carlo simulation method of 500 times, compared to the MVM and MUSIC algorithm for the nonplanar quaternion array, The SNR is fixed at 15, and the number of snapshots range from 50 to 500 . Notice that the deviation and variance become smaller along with the increase of the number of snapshots for the nonplanar quaternion array. Notice that the performance of the MUSIC algorithm is poor, when the number of snapshots is less than 500. In contrast, the performance of the MVM algorithm and m-MVM algorithm are better for the number of snapshots more than 200 .

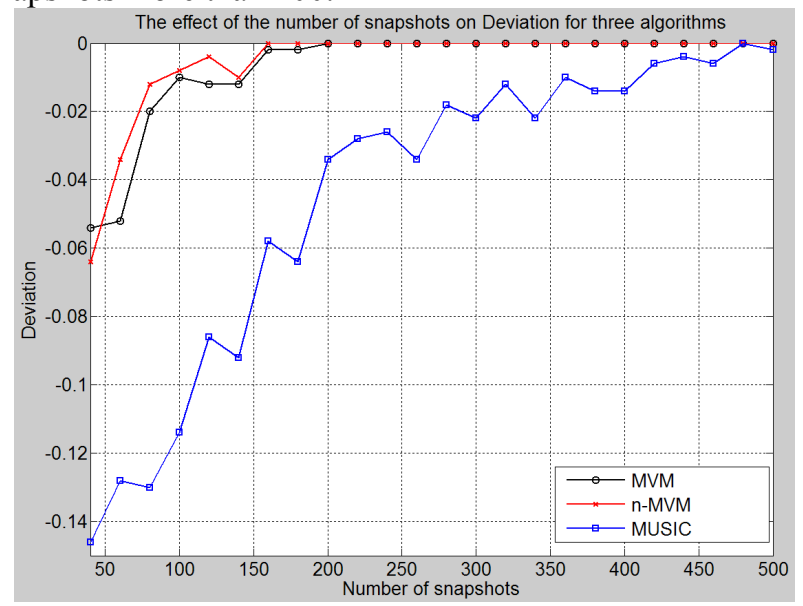

Figure 7. The effect of the number of snapshots on Deviation for the three algorithms

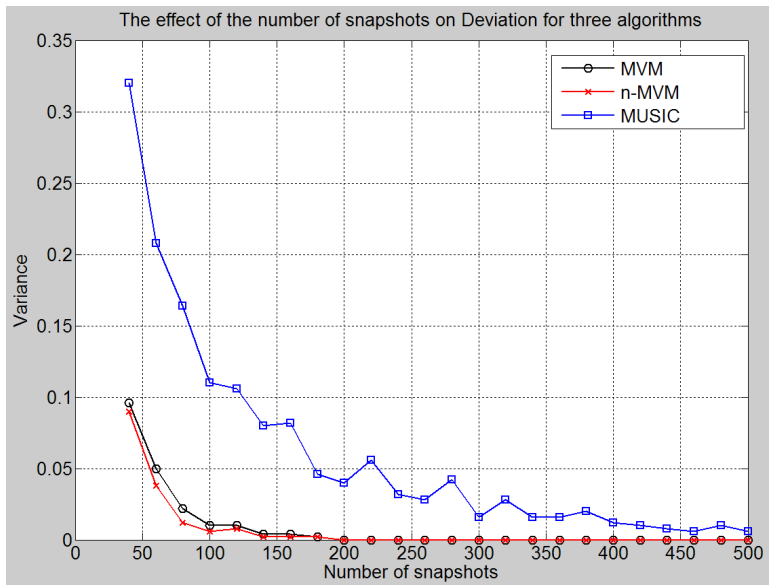

Figure 8. The effect of the number of snapshots on variance for the three algorithms

Figure 9 and Figure 10 shows the variance of the m-
MVM algorithm by Monte Carlo simulation method of 1000 times, compared to the MVM and MUSIC algorithm for the uniform cross array and the nonplanar quaternion array, The number of snapshots is fixed at 256, and the SNR range from $-5 \mathrm{~dB}$ to $20 \mathrm{~dB}$. Notice that the variance becomes smaller along with the increase of SNR for the nonplane quaternion array and uniform cross array. The variance of the m-MVM algorithm is smaller than the MVM algorithm, and the variance of the MUSIC algorithm is the least.

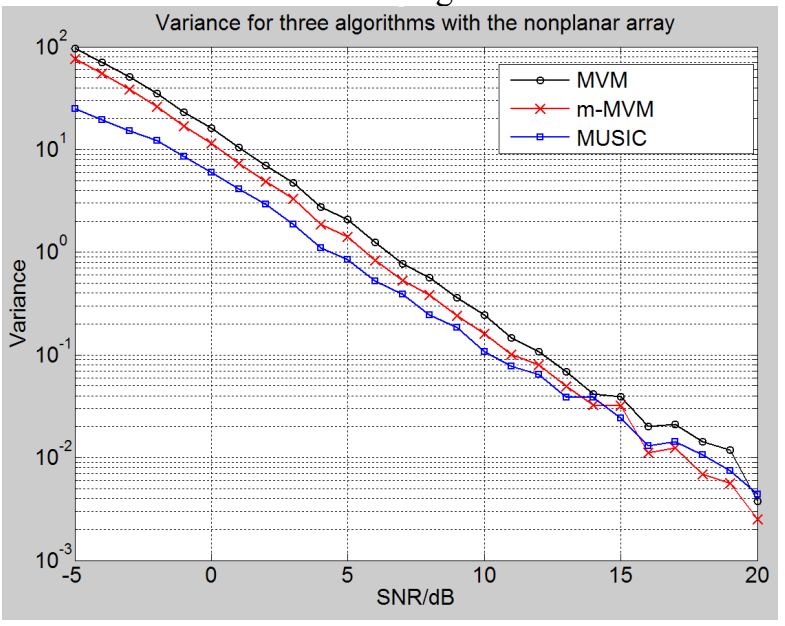

Figure 9. The effect of SNR on variance for three algorithms with nonplanar array

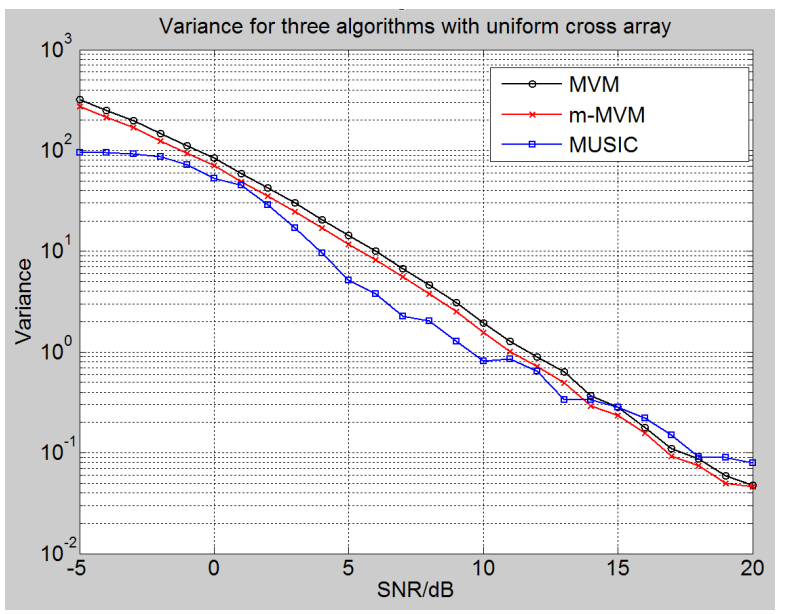

Figure 10. The effect of SNR on variance for three algorithms with uniform cross array

Figure 11 shows the effect of SNR on variance using the m-MVM algorithm with uniform cross array by Monte Carlo simulation method of 1000 times, compared to the nonplanar quaternion array, The number of snapshots is fixed at $256, \mathrm{SNR} \in[-5 \mathrm{~dB}, 20 \mathrm{~dB}]$. Notice that the variance of the nonplanar quaternion array is smaller than planar array. The variance becomes smaller along with the increase of SNR for the nonplanar quaternion array and the uniform cross array. 


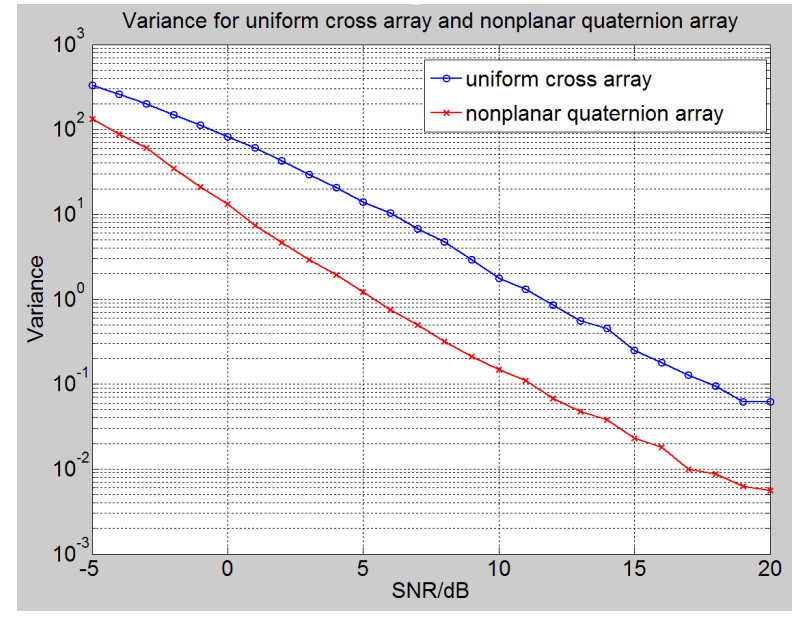

Figure 11. The effect of SNR on variance for uniform cross array and nonplanar quaternion array

\section{CONCLUSIONS}

In sensor array processing, most high-resolution algorithms depend on the source number estimation, the performance of direction-finding decreases significantly with underestimation or overestimation of the source number. In this paper, an m-MVM algorithm without source number estimation and eigendecomposition is proposed for nonplanar array with arbitrary geometry. Numerical examples demonstrate the validity of the proposed algorithm, and some conclusions of the proposed algorithm for the nonplanar quaternion array and planar array are reached.

\section{ACKNOWLEDGMENT}

The authors wish to thank Prof. Xiang and the anonymous reviewers for their suggestions and comments which contributed to the final form of the paper.

\section{REFERENCES}

[1] Pascal Chevalier, Anne Ferréol, and Laurent Albera."High-Resolution Direction Finding From Higher Order Statistics: The 2q-MUSIC Algorithm", IEEE Transactions on signal processing, vol. 54, no. 8, August 2006

[2] Zhu Weiqing, Hu Juan, Liu Xiaodong, Liu Zhiyu and Zhu Min. "Source number estimation using eigenspace in direction of arrival (DOA) estimate" OCEANS 2009-EUROPE, 2009. OCEANS '09.1114 May 2009 Page(s):1-6

[3] Lu Pin and Zhang Zhijun. "A Performance Analys is of the Modified MUSIC Algorithm Without Considering the Source Number" Control \& Automation, Vol. 23, No. 30, 2007

[4] Zhang Zhi-jun, Hou Zhi-bin and Zhang Hao "A Modified MUSIC Algorithm without Considering the Source Number" Telecommunication Engineering, Vol. 47, No. 5, Oct. 2007

[5] Du Jingjing, Zhang Yang and Tang Bin. "Estimation of blind source number in communication reconnaissance" Signal Processing, 2008. ICSP 2008. 9th International Conference on 26-29 Oct. 2008 Page(s):1815-1817

[6] Qingchun Zhang and Zhenghe Feng. "A Novel Source Number Estimator Without Eigendecomposition" Antennas, Propagation \& EM Theory, 2006. ISAPE '06. 7th International Symposium on 26-29 Oct. 2006 Page(s): $1-3$

[7] Radich, B.M.and Buckley, K.M. "The effect of source number underestimation on MUSIC location estimates" Signal Processing, IEEE Transactions on [see also Acoustics, Speech, and Signal Processing, IEEE Transactions on Volume 42, Issue 1, Jan. 1994 Page(s):233-236

[8] Qiao-wei Yuan, Qiang Chen, Member and Kunio Sawaya. "Accurate DOA Estimation Using Array Antenna With Arbitrary Geometry" IEEE Transactions on antennas and propagation, vol. 53, NO. 4, april 2005. 